\title{
Gamma-ray observations of W44 and its surroundings
}

\section{Giada Peron, ${ }^{a, *}$ Felix Aharonian, ${ }^{a, b}$ Sabrina Casanova, ${ }^{a, c}$ Roberta Zanin ${ }^{e}$ and Carlo Romoli $^{a}$}

${ }^{a}$ Max-Planck-Institut für Kernphysik, Saupfercheckweg 1, Heidelberg, Germany

${ }^{b}$ Dublin Institute for Advanced Studies, 31 Fitzwilliam Place, Dublin 2, Ireland

${ }^{c}$ Institute of Nuclear Physics PAN,

Radzikowskiego 152, 31-342 Kraków, Poland

${ }^{d}$ CTA observatory,

Via Piero Gobetti 93/3 40129 Bologna, Italy

E-mail: giada.peron@mpi-hd.mpg.de

We present the analysis of 9.7 years Fermi-LAT data of the middle-aged supernova remnant (SNR) W44 and the massive molecular gas complex that surrounds it. The derived spectral energy distribution of the SNR, derived over three decades is improved, with respect to previous observations, both at low $(<100 \mathrm{MeV})$ and at higher energies $(>100 \mathrm{GeV})$ allowing us to strongly constrain the hadronic origin of the emission. We also unveil the presence of two extended $\gamma$-ray structures located at two opposite edges of the remnant along its major axis. These two sources do not coincide with any peak in the gas distribution, therefore are interpreted as "CR clouds", namely as regions of enhanced CR density, consisting of particles that escaped collectively from the remnant along the magnetic field.

$37^{\text {th }}$ International Cosmic Ray Conference (ICRC 2021)

July 12 th $-23 r d, 2021$

Online - Berlin, Germany

\footnotetext{
${ }^{*}$ Presenter
} 


\section{Introduction}

Supernova remnants (SNRs) have been proven to be effective accelerators both at $\mathrm{GeV}$ and $\mathrm{TeV}$ energies. However, even if their power seems sufficient to support the entire population of Galactic cosmic rays, so far there has been no observational evidence that these kind of sources are able to accelerate particles up to a few $\mathrm{PeV}$, which is the highest energy end of the galactic $\mathrm{CR}$ spectrum. Recent observations published by LHAASO [2] effectively proved the existence of PeV accelerators in the Galaxy, but the identification of these accelerators is still unclear. The theory of diffusive shock acceleration (DSA) applied to supernova remnants suggests that even if SNRs were effectively PeVatrons, they would be PeVatrons only for a short period of time [3], making high energy emission harder to detect. As the shock slows down, the highest energy particles are no longer confined and start to leave the remnant. The runaway particles populate the surroundings and produce detectable $\gamma$-emission. The simultaneous study of the emission both from the remnant and the surrounding medium, allows us to reconstruct not only the current, but also the precedent acceleration power of the remnant and thus to clarify which energy these accelerator can reach [5].

We chose as our target the SNR W44, which is a middle-age supernova remnant of age 10000 yr. At this age, the SNR is already in the Sedov phase, where the shock has already started to slow down and the accelerated particles have started to leave the remnant. Moreover, W44 is embedded in a massive $\left(\sim 10^{6} \mathrm{M}_{\odot}\right)$ molecular cloud complex, enhancing the chance of detection of gamma-ray emission.

\section{Observations}

We analyzed Fermi-LAT Pass8 data in a $10^{\circ}$ region centered on $\mathrm{W} 44$, at $(1, \mathrm{~b})=(34.6,-0.36)^{\circ}$. We used both FRONT and BACK reconstructed events (evtype $=3$ ), and imposed the standard quality cuts $\left(\mathrm{z}_{\max }=90^{\circ}\right.$, and DATA_QUAL $==1 \&$ LAT_CONFIG $\left.==1\right)$. To model both the galactic and the extragalactic diffuse component, we used the latest released templates provided by the Fermi-LAT collaboration ${ }^{1}$. We included in the model, also all the sources from the fourth FermiLAT catalog (4FGL;[? ]). The morphology and the spectrum of the closest 4FGL sources $\left(\lesssim 1^{\circ}\right)$ to the remnants were remodeled before modeling the spatial and spectral distributions of the remnant and the surrounding region.

Different morphological and spectral models were compared, based on the Aikake information criterion (AIC). The preferred shape turned out to be an elliptical ring, with the major axis aligned with the magnetic field lines. A similar morphology is also observed in radio and it was suggested to be related to interaction of the remnant with the surrounding medium [9]. The preferred spectral model turned out to be a $\log$ parabola: $d N / d E=N_{0}\left(E / E_{b}\right)^{-\left(\alpha+\beta \log \left(E / E_{b}\right)\right)}$, with the last detected points around $100 \mathrm{GeV}$ (Fig 1). The spectrum has a steep falloff at high energies, where the spectral index is $\sim 3.3$. This is consistent with the hypothesis hat particles of energies exceeding a few $\mathrm{TeV}$ have already left the remnant. The SED of the remnant is well modeled both by an hadronic (pion decay) and by a leptonic (bremsstrahlung) scenario. In the first case, the pion bump feature naturally accounts for the flattening at a few $\mathrm{GeV}$, and a cutoff at $\sim 40 \mathrm{GeV}$ is needed to model the high energy steepening. In the second case, an additional low energy break of the electron spectrum

\footnotetext{
${ }^{1}$ https://fermi.gsfc.nasa.gov/ssc/data/access/lat/BackgroundModels.html
} 

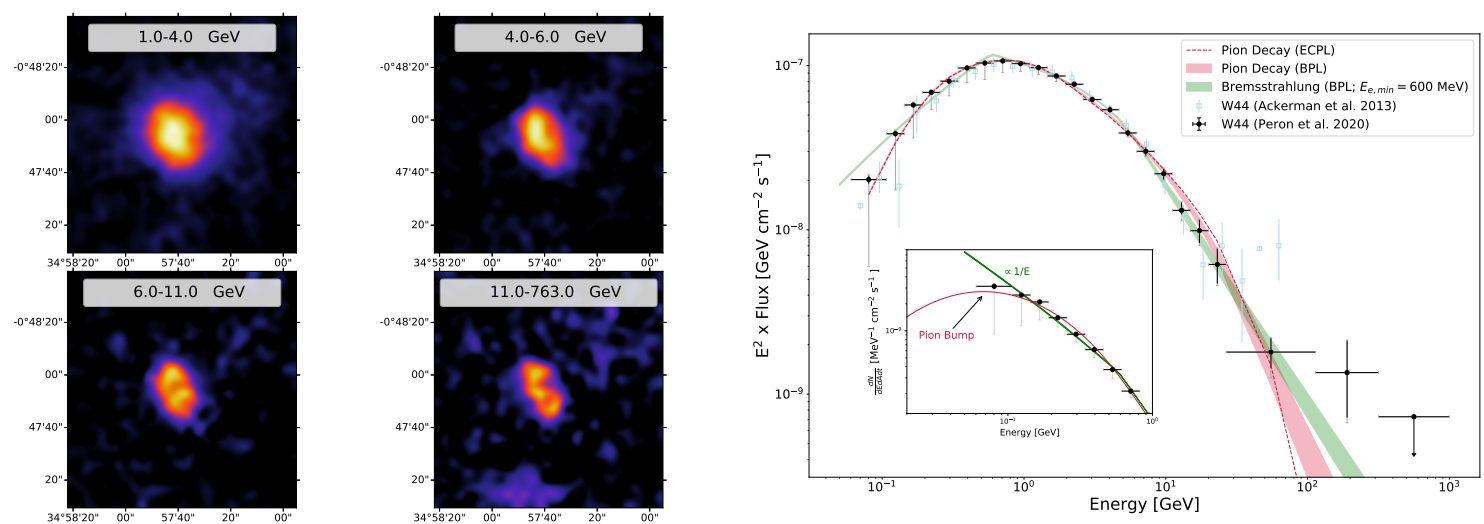

Figure 1: On the left: counts maps of the W44 for different energy bins after the subtraction of the other sources. On the right: the SED extracted from the region of W44. The red and the green curves show the best-fit hadronic and leptonic model respectively.

(at $600 \mathrm{MeV}$ ) is needed to account for the low energy part of the spectrum. Even so, the resulting spectrum below the break would have a $E^{-1}$ dependence, which is not consistent with our spectral measurements. Despite the large uncertainties of the spectral points below $1 \mathrm{GeV}$, the pion decay is preferred over the bremsstrahlung.

After modeling the remnant we investigated the emission from the surrounding medium. Two extended sources $\left(0.4^{\circ}\right.$ and $\left.0.15^{\circ}\right)$ appeared at the opposite edges of the remnant, along the major axis (see Figure 2). For both sources, the extended disk morphology was preferred over a set of single point sources, as reported in the 4FGL catalog. We named the two sources NW-source and SE-source as they are located in the north western part and in the southern east part of the ROI. The northern cloud has a larger area than the southern one and its spectrum is also harder and extends to higher energies (see Figure 3). The spectrum of the northern cloud is harder also compared to the remnant, consistent with the idea that this region hosts the runaway particles that already left the remnant. The spectrum of the southern cloud is similar to the spectrum of the SNR but somewhat shifted towards higher energies. The sharp drop of its spectrum, however, indicates that in these region, the highest energy particle are not retained.

\section{Gas analysis}

We compared the $\gamma$-ray observations with the gas distribution from the FOREST Unbiased Galactic plane Imaging survey with the Nobeyama 45-m telescope (FUGIN) of ${ }^{12} \mathrm{CO}(\mathrm{J}=1 \rightarrow 0)$ line emission. The analysis was restricted to gas in the $30-65 \mathrm{~km} \mathrm{~s}^{-1}$ range. W44 radial velocity was determined from masers measurements to be $45 \mathrm{~km} \mathrm{~s}^{-1}$ [6]. The region contains several molecular clouds with masses $0.3-3 \times 10^{5} \mathrm{M}_{\odot}$. However there is not exact correspondence between the region of the $\gamma$-ray emission and the location of any of these clouds. SE-source partly coincide with GMC G34.6-0.7 while NW-source is at the edge of GMC G35.0+0.3. Interesting to notice, no $\gamma$-ray emission is reveiled in the region of GMC G34.8-0.5, which is more massive and closer than the other cloud regions. The gas density in the region where we detect $\gamma$-ray emission is instead close to the average $\sim 10 \mathrm{~cm}^{-3}$. We calculated the gas mass included in the regions of SE-source and 

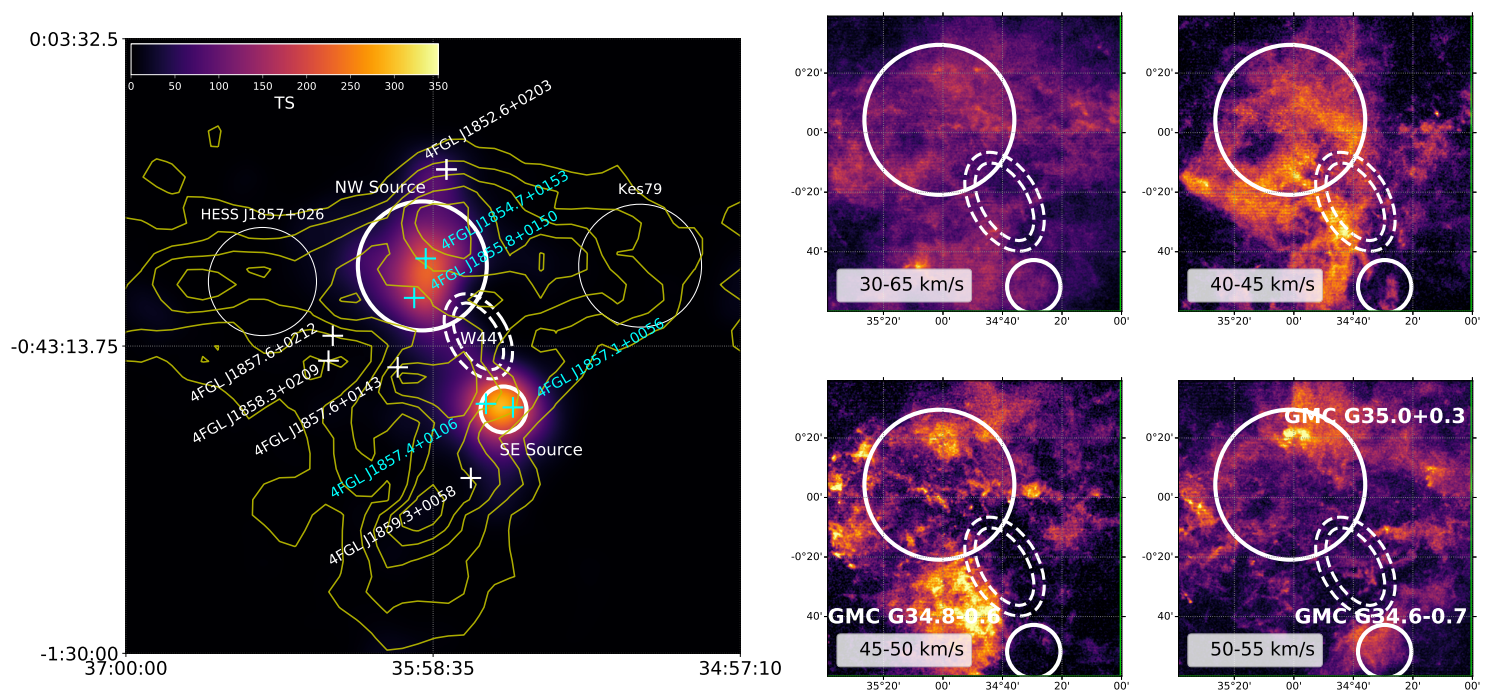

Figure 2: On the left: the TS map of the two extended sources. The white regions are the sources in the model, the cyan regions are the sources which have been deleted. The yellow contours trace the CO gas of [4]. On the right: the distribution of the $\mathrm{CO}$ gas in different velocity ranges from the FUGIN survey [10]
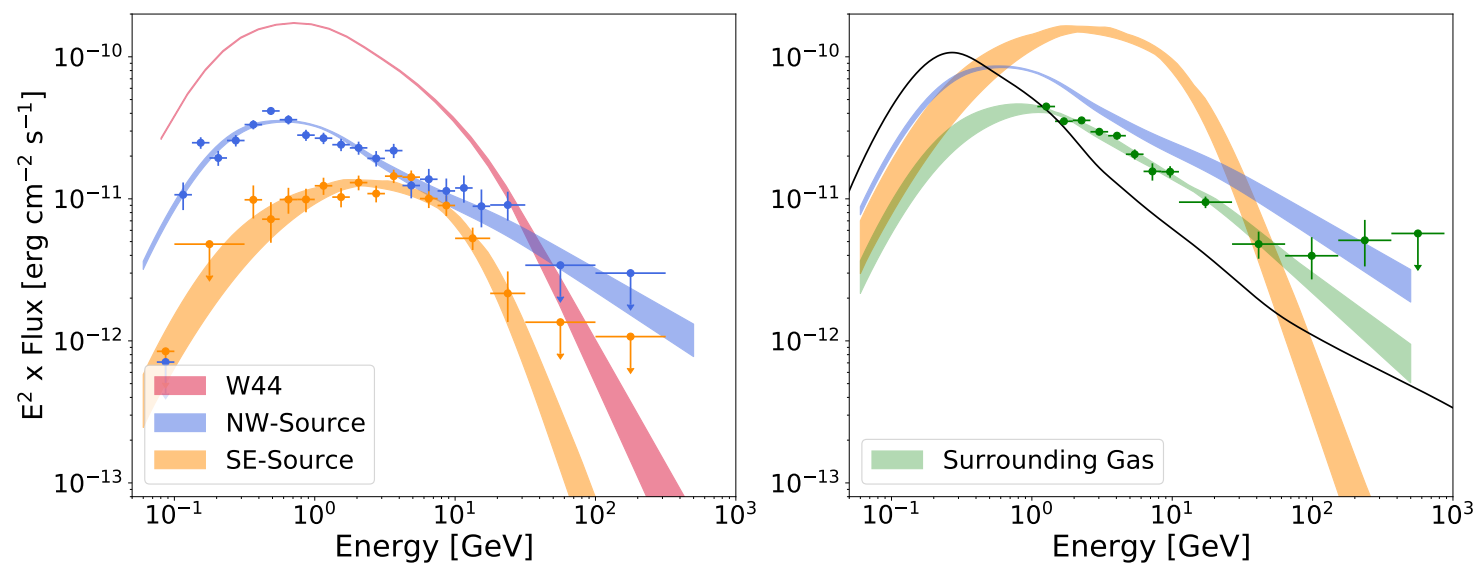

Figure 3: On the left: the SED of the two sources are compared to the spectrum of the remnant. On the right: the SED of the clouds and of the entire molecular cloud complex, normalized to $M_{5}=d_{k p c}=1$. The $\gamma$-ray emissivity of a cloud illuminated by a local spectrum of CRs is also plotted in black for comparison. Figure adapted from [8]

NW-source from CO observations and it resulted $0.4 \times 10^{5} \mathrm{M}_{\odot}$ and $2 \times 10^{5} \mathrm{M}_{\odot}$. This means that the $\gamma$-ray emission is due to an enhance CR density rather than a enhanced gas density. The normalized for mass , $M_{5} \equiv M /\left(10^{5} \mathrm{M}_{\odot}\right)=1$, and distance, $d_{k p c}=d /(1 \mathrm{kpc})=1$, SEDs are shown in the right panel of Figure 3. The derived flux are compared to the flux expected from a molecular cloud illuminated by the local CR spectrum. Both sources have a largely enhanced flux by a factor $\sim 3$ and $\sim 7$ for the northern and southern region respectively and the spectral shape is also not compatible.

As a further step, we examined the gamma-ray signal arising from the entire gas region. The 
analysis was conducted following the analysis technique of [1]. The resulting spectrum has a similar shape as the local one and it is enhance only of a factor $\sim 50 \%$, which is compatible with the uncertainties on the mass of the gas. This further support the idea that the enhacement is localized only in two extended regions.

\section{Conclusion}

The re-analysis of the SNR W44 provided further and stronger evidence for the hadronic origin of the emission. Moreover, the analysis of the surrounding $\gamma$-ray emission, combined with the analysis of the gas unveiled that the emission is not originating in regions of enhanced gas density, but then should be due to localized enhanced CR density in sort of "CR clouds". The collective escape of particles in the direction of the field lines was already predicted by Malkov et al. [7]. Although the differences in the morphology and spectra of the two clouds still need to be clarified, this might be the first detection of anisotropic escape from a supernova remnant along the magnetic field.

\section{References}

[1] Felix Aharonian, Giada Peron, Ruizhi Yang, Sabrina Casanova, and Roberta Zanin. Probing the sea of galactic cosmic rays with Fermi-LAT. Physical Review D, 101(8), 2020.

[2] Zhen Cao, F. A. Aharonian, Q. An, Axikegu, L. X. Bai, Y. X. Bai, Y. W. Bao, D. Bastieri, X. J. Bi, Y. J. Bi, H. Cai, J. T. Cai, Zhe Cao, J. Chang, J. F. Chang, X. C. Chang, B. M. Chen, J. Chen, L. Chen, Liang Chen, Long Chen, M. J. Chen, M. L. Chen, Q. H. Chen, S. H. Chen, S. Z. Chen, T. L. Chen, X. L. Chen, Y. Chen, N. Cheng, Y. D. Cheng, S. W. Cui, X. H. Cui, Y. D. Cui, B. Z. Dai, H. L. Dai, Z. G. Dai, Danzengluobu, D. della Volpe, B. DEttorre Piazzoli, X. J. Dong, J. H. Fan, Y. Z. Fan, Z. X. Fan, J. Fang, K. Fang, C. F. Feng, L. Feng, S. H. Feng, Y. L. Feng, B. Gao, C. D. Gao, Q. Gao, W. Gao, M. M. Ge, L. S. Geng, G. H. Gong, Q. B. Gou, M. H. Gu, J. G. Guo, X. L. Guo, Y. Q. Guo, Y. Y. Guo, Y. A. Han, H. H. He, H. N. He, J. C. He, S. L. He, X. B. He, Y. He, M. Heller, Y. K. Hor, C. Hou, X. Hou, H. B. Hu, S. Hu, S. C. Hu, X. J. Hu, D. H. Huang, Q. L. Huang, W. H. Huang, X. T. Huang, Z. C. Huang, F. Ji, X. L. Ji, H. Y. Jia, K. Jiang, Z. J. Jiang, C. Jin, D. Kuleshov, K. Levochkin, B. B. Li, Cong Li, Cheng Li, F. Li, H. B. Li, H. C. Li, H. Y. Li, J. Li, K. Li, W. L. Li, X. Li, Xin Li, X. R. Li, Y. Li, Y. Z. Li, Zhe Li, Zhuo Li, E. W. Liang, Y. F. Liang, S. J. Lin, B. Liu, C. Liu, D. Liu, H. Liu, H. D. Liu, J. Liu, J. L. Liu, J. S. Liu, J. Y. Liu, M. Y. Liu, R. Y. Liu, S. M. Liu, W. Liu, Y. N. Liu, Z. X. Liu, W. J. Long, R. Lu, H. K. Lv, B. Q. Ma, L. L. Ma, X. H. Ma, J. R. Mao, A. Masood, W. Mitthumsiri, T. Montaruli, Y. C. Nan, B. Y. Pang, P. Pattarakijwanich, Z. Y. Pei, M. Y. Qi, D. Ruffolo, V. Rulev, A. Sáiz, L. Shao, O. Shchegolev, X. D. Sheng, J. R. Shi, H. C. Song, Yu V. Stenkin, V. Stepanov, Q. N. Sun, X. N. Sun, Z. B. Sun, P. H.T. Tam, Z. B. Tang, W. W. Tian, B. D. Wang, C. Wang, H. Wang, H. G. Wang, J. C. Wang, J. S. Wang, L. P. Wang, L. Y. Wang, R. N. Wang, W. Wang, W. Wang, X. G. Wang, X. J. Wang, X. Y. Wang, Y. D. Wang, Y. J. Wang, Y. P. Wang, Zheng Wang, Zhen Wang, Z. H. Wang, Z. X. Wang, D. M. Wei, J. J. Wei, Y. J. Wei, T. Wen, C. Y. Wu, H. R. Wu, S. Wu, W. X. Wu, X. F. Wu, 
S. Q. Xi, J. Xia, J. J. Xia, G. M. Xiang, G. Xiao, H. B. Xiao, G. G. Xin, Y. L. Xin, Y. Xing, D. L. Xu, R. X. Xu, L. Xue, D. H. Yan, C. W. Yang, F. F. Yang, J. Y. Yang, L. L. Yang, M. J. Yang, R. Z. Yang, S. B. Yang, Y. H. Yao, Z. G. Yao, Y. M. Ye, L. Q. Yin, N. Yin, X. H. You, Z. Y. You, Y. H. Yu, Q. Yuan, H. D. Zeng, T. X. Zeng, W. Zeng, Z. K. Zeng, M. Zha, X. X. Zhai, B. B. Zhang, H. M. Zhang, H. Y. Zhang, J. L. Zhang, J. W. Zhang, L. Zhang, Li Zhang, L. X. Zhang, P. F. Zhang, P. P. Zhang, R. Zhang, S. R. Zhang, S. S. Zhang, X. Zhang, X. P. Zhang, Yong Zhang, Yi Zhang, Y. F. Zhang, Y. L. Zhang, B. Zhao, J. Zhao, L. Zhao, L. Z. Zhao, S. P. Zhao, F. Zheng, Y. Zheng, B. Zhou, H. Zhou, J. N. Zhou, P. Zhou, R. Zhou, X. X. Zhou, C. G. Zhu, F. R. Zhu, H. Zhu, K. J. Zhu, and X. Zuo. Ultrahigh-energy photons up to 1.4 petaelectronvolts from $12 \gamma$-ray Galactic sources. Nature, 594(7861):33-36, jun 2021.

[3] Pierre Cristofari, Pasquale Blasi, and Elena Amato. The low rate of Galactic pevatrons. Astroparticle Physics, 123:102492, dec 2020.

[4] T M Dame, Dap Hartmann, and P Thaddeus. The Milky Way in Molecular Clouds: A New Complete CO Survey. Technical report.

[5] Stefano Gabici, Felix A. Aharonian, and Pasquale Blasi. Gamma rays from molecular clouds. Astrophysics and Space Science, 309(1-4):365-371, jun 2007.

[6] Ian M. Hoffman, W. M. Goss, C. L. Brogan, and M. J. Claussen. The OH (1720 MHz) Supernova Remnant Masers in W44: MERLIN and VLBA Polarization Observations. The Astrophysical Journal, 627(2):803-812, jul 2005.

[7] M A Malkov, P H Diamond, R Z Sagdeev, F A Aharonian, and I V Moskalenko. ANALYTIC SOLUTION FOR SELF-REGULATED COLLECTIVE ESCAPE OF COSMIC RAYS FROM THEIR ACCELERATION SITES. The Astrophysical Journal, 768(13pp):73, 2013.

[8] Giada Peron, Felix Aharonian, Sabrina Casanova, Roberta Zanin, and Carlo Romoli. On the Gamma-Ray Emission of W44 and Its Surroundings. The Astrophysical Journal Letters, 896(2), 2020.

[9] Masumichi Seta, Tetsuo Hasegawa, T. M. Dame, Seiichi Sakamoto, Tomoharu Oka, Toshihiro Handa, Masahiko Hayashi, Jun-Ichi Morino, Kazuo Sorai, and Kumiko S. Usuda. Enhanced $\mathrm{CO} \mathrm{J}=2-1 / \mathrm{J}=1-0$ Ratio as a Marker of Supernova Remnant-Molecular Cloud Interactions: The Cases of W44 and IC 443 . The Astrophysical Journal, 505(1):286-298, sep 1998.

[10] T Umemoto, T Minamidani, and N Kuno. FOREST unbiased Galactic plane imaging survey with the Nobeyama $45 \mathrm{~m}$ telescope (FUGIN). I. Project overview and initial results. Publications of the Astronomical Society of Japan, 69, 2017. 